\title{
Flavour improvement of reduced-fat peanut butter by addition of a kokumi peptide, Y-glutamyl-valyl-glycine
}

\author{
Naohiro Miyamura ${ }^{1}$, Shuichi Jo ${ }^{1}$, Motonaka Kuroda $^{1 *}$ and Tohru Kouda ${ }^{2}$
}

\begin{abstract}
Background: Recent studies have demonstrated that kokumi substances, which enhance basic tastes and modify mouthfulness and continuity although they have no taste themselves, are perceived through the calcium-sensing receptor (CaSR). Screening by a CaSR assay and sensory evaluation have shown that $\gamma$-glutamyl-valyl-glycine ( $\gamma$-Glu-Val-Gly) is a potent kokumi peptide. In our previous study, it was reported that the addition of $\gamma$-Glu-Val-Gly to chicken consommé significantly enhanced mouthfulness, continuity and thickness. In this study, the effect of Y-Glu-Val-Gly on reduced-fat peanut butter was investigated.

Results: Prior to the evaluation of the effect of $\gamma$-Glu-Val-Gly, a comparison test was conducted between full-fat model peanut butter and reduced-fat peanut butter. The sensory attributes in which the score of the full-fat model was significantly higher than that of the reduced-fat sample were used for the evaluation of the effect of $\gamma$-Glu-Val-Gly. The addition of $\gamma$-Glu-Val-Gly significantly enhanced thick flavour, aftertaste, and oiliness in the reduced-fat peanut butter.
\end{abstract}

Conclusions: A kokumi peptide, $\gamma$-Glu-Val-Gly, can enhance thick flavour, aftertaste and oiliness in reduced-fat peanut butter. This suggests that addition of $\gamma$-Glu-Val-Gly can improve the flavour of low-fat foods.

Keywords: Low-fat foods, Reduced-fat foods, Peanut butter, Kokumi, y-glutamyl-valyl-glycine, Sensory evaluation

\section{Background}

Recent studies have revealed that kokumi substances such as glutathione (GSH) are perceived through the calciumsensing receptors (CaSRs) in humans [1]. These studies have confirmed that GSH can activate human CaSRs, as can several $\gamma$-glutamyl peptides, including $\gamma$-Glu-Ala, $\gamma$-Glu-Val, $\gamma$-Glu-Cys, $\gamma$-Glu- $\alpha$-aminobutyryl-Gly (ophthalmic acid) and $\gamma$-Glu-Val-Gly. Furthermore, these compounds have been shown to possess the characteristics of kokumi substances, which modify the five basic tastes (especially sweet, salty and umami) when added to basic taste solutions or food, even though they have no taste themselves at the concentrations tested [2-8]. The CaSR activity of these $\gamma$-glutamyl peptides is positively correlated with the sensory activity of kokumi substances, suggesting they are perceived through the CaSRs in humans. Among these,

\footnotetext{
* Correspondence: motonaka_kuroda@ajinomoto.com

${ }^{1}$ Institute of Food Research and Technologies, Ajinomoto Co. Inc., 1-1

Suzuki-cho, Kawasaki-ku, Kawasaki, Kanagawa 210-8681, Japan

Full list of author information is available at the end of the article
}

$\gamma$-Glu-Val-Gly has been reported to be a potent kokumi peptide with a sensory activity 12.8 -fold greater than that of GSH [3].

In our previous study, the effect of $\gamma$-Glu-Val-Gly on the sensory characteristics of chicken consommé was investigated. Adding $\gamma$-Glu-Val-Gly to chicken consommé significantly enhanced thickness (taste enhancement $\sim 5 \mathrm{~s}$ after tasting), continuity (taste intensity at $20 \mathrm{~s}$ after tasting), and mouthfulness (the reinforcement of taste sensation throughout the mouth and not just on the tongue) [3]. It is generally known that these sensations are evoked by the addition of fat-containing food materials such as dairy fat emulsion [9].

The problem of the increase in the obese population has resulted in various kinds of reduced-fat foods being developed and commercialised. However, in general, the palatability of reduced-fat foods is lower than that of full-fat foods. In previous studies, it has been demonstrated that the reduced-fat samples have decreased juiciness, greasiness, aftertaste, and overall flavour intensity in 
sausages [10] and decrease the score of creaminess in yogurt [11]. In addition, it has been demonstrated that the reduced-fat samples have lower scores in thickness, smoothness, creaminess, mouth coating and milky/cooked sugar flavour in ice cream [12] and have lower scores in milk fat flavour and brothy flavour in cheddar cheese [13]. To overcome these problems, because reduced-fat foods mainly lack texture, the use of thickeners such as gums, starch and modified starch has been proposed. However, the reduced-fat foods with such additives still have lower palatability than full-fat foods.

In the present study, we aimed to clarify whether addition of $\gamma$-Glu-Val-Gly changed the flavour by palatability of reduced-fat foods. We investigated the effect of $\gamma$-Glu-Val-Gly on reduced-fat peanut butter.

\section{Results and discussion}

In this study, first, the sensory attributes of peanut butter were discussed and selected by expert panellists. Then, panellists rated the differences between reducedand full-fat peanut butter to establish how increased fat affected the sensory attributes of peanut butter. Finally, the same evaluation was conducted comparing reducedfat peanut butter and that with kokumi peptide, $\gamma$-GluVal-Gly.

\section{Sensory attributes}

During the group discussion, panellists listed up the words, selected the attributes and made a consensus of the sensation which the attribute expressed. Finally, the panellists developed ten attributes: peanut flavour, saltiness, sweetness, bitterness, thick flavour (thickness of taste; the enhancement of taste intensity with maintaining the balance of taste), aftertaste (the total aftertaste intensity after $5 \mathrm{~s}$ of all flavour notes within the sample), continuity of taste (the taste intensity at $\sim 20 \mathrm{~s}$ ), smoothness, and oiliness.

\section{Comparison between reduced-fat sample and full-fat model of peanut butter}

Comparison between reduced-fat peanut butter and full-fat model peanut butter is shown in Table 1. The full-fat model peanut butter had higher scores for peanut flavour, thick flavour, aftertaste, continuity of taste, and oiliness than for low-fat peanut butter. No significant difference in saltiness, sweetness, bitterness, smoothness, and viscous sensation was observed between the low-fat sample and full-fat model. We consider that the fat enhanced the above sensory character in peanut butter. In other words, we considered that peanut flavour, thick flavour, aftertaste, continuity of taste, and oiliness were the sensory functions of fat in peanut butter.
Table 1 Result of the comparison test between low-fat peanut butter and full-fat model peanut butter

\begin{tabular}{lcl}
\hline Sensory attributes & Score of full-fat model & Significance \\
\hline Peanut flavour & $0.24 \pm 0.05$ & $* *$ \\
Saltiness & $0.04 \pm 0.04$ & NS \\
Sweetness & $-0.03 \pm 0.05$ & NS \\
Bitterness & $-0.10 \pm 0.05$ & NS \\
Thick flavour & $0.15 \pm 0.06$ & $*$ \\
Aftertaste & $0.16 \pm 0.04$ & $* *$ \\
Continuity of taste & $0.14 \pm 0.04$ & $* *$ \\
Smoothiness & $0.10 \pm 0.08$ & NS \\
Viscosity & $-0.01 \pm 0.09$ & NS \\
Oiliness & $0.23 \pm 0.07$ & $* *$
\end{tabular}

Description of data: data was shown as means \pm standard errors. Abbreviation: NS not significant. ${ }^{*} p<0.05 ;{ }^{* *} p<0.01$.

\section{Effect of addition of $\gamma$-Glu-Val-Gly in reduced-fat peanut butter}

To clarify the effect of $\gamma$-Glu-Val-Gly on the sensory character of reduced-fat peanut butter, reduced-fat peanut butter with $40 \mathrm{ppm} \gamma$-Glu-Val-Gly was evaluated for the attributes, peanut flavour, thick flavour, aftertaste, continuity of taste, and oiliness. The results of the sensory evaluation are shown in Table 2. Addition of $\gamma$-GluVal-Gly significantly enhanced the intensities of thick flavour, aftertaste, and oiliness. These results demonstrated that the addition of $\gamma$-Glu-Val-Gly increased some sensations that were lacking in the reduced-fat peanut butter, suggesting that addition of the peptide can be used for flavour improvement in reduced-fat peanut butter.

The previous studies described that the several reducedfat foods and low-fat foods lacked the sensations related to 'thick flavour', 'aftertaste' and 'oiliness'. For example, it has been previously reported that the low-fat sausage has lower juiciness and aftertaste intensity [10] and that lowfat yogurt has lower creaminess [11] than full-fat products. In addition, it has been previously reported that reducedfat ice cream indicated lower scores of texture-related attributes such as thickness, smoothness, creaminess,

Table 2 Effect of $y$-Glu-Val-Gly on the low-fat peanut butter

\begin{tabular}{lcl}
\hline Sensory attributes & $\begin{array}{c}\text { Score of sample with } \\
\text { p-Glu-Val-Gly }\end{array}$ & Significance \\
\hline Peanut flavour & $0.06 \pm 0.05$ & NS \\
Thick flavour & $0.13 \pm 0.04$ & $* *$ \\
Aftertaste & $0.14 \pm 0.05$ & $*$ \\
Continuity of taste & $0.09 \pm 0.05$ & NS \\
Oiliness & $0.09 \pm 0.04$ & $*$
\end{tabular}

Data was shown as means \pm standard errors.

Abbreviation: NS not significant.

${ }^{*} p<0.05 ;{ }^{* *} p<0.01$. 
mouth coating than those of full-fat products [12]. Therefore, it is considered that the addition of $\gamma$-Glu-Val-Gly can be used to improve the flavour of other reduced-fat foods. In order to clarify this possibility, it is necessary to conduct a preference test using a consumer panel, and this test is now in progress in our laboratory. The effect of $\gamma$-Glu-Val-Gly on other reduced-fat foods is also now under investigation in our laboratory.

\section{Conclusions}

In this study, the effect of a kokumi peptide, $\gamma$-Glu-ValGly, on the flavour of reduced-fat peanut butter was investigated. The results indicated that the addition of $\gamma$-Glu-Val-Gly significantly enhanced the intensities of thick flavour, aftertaste, and oiliness. These results demonstrated that addition of $\gamma$-Glu-Val-Gly increased some sensations that were lacking in the reduced-fat peanut butter, suggesting that addition of the peptide could improve the flavour of reduced-fat peanut butter.

\section{Methods}

\section{Preparation of $\gamma$-Glu-Val-Gly}

The $\gamma$-Glu-Val-Gly used in the present study was of food additive grade (FEMA-GRAS No. 4709; Flavor and Extract Manufacturers Association (FEMA); JECFA food flavouring No. 2123; Joint FAO/WHAO Expert Committee on Food Additives (JECFA)) obtained from Ajinomoto Co. Inc. (Tokyo, Japan) and was prepared by chemical synthesis as reported previously [1].

\section{Preparation of reduced-fat peanut butter and full-fat peanut butter model}

The raw materials for reduced-fat peanut butter $(30 \%$ fat content) and full-fat peanut butter model (50\% fat content) are shown in Table 3. Regarding the preparation of the reduced-fat peanut butter, the emulsifiers were mixed with peanut paste in an aluminium pot at $30^{\circ} \mathrm{C}$ with later addition of cream by stirring. Sugar and salt solubilised in

Table 3 Raw materials for the low-fat peanut butter and full-fat model peanut butter

\begin{tabular}{lcc}
\hline Materials & $\begin{array}{c}\text { Low-fat } \\
\text { (wt.\%) }\end{array}$ & $\begin{array}{c}\text { Full-fat } \\
\text { model (wt.\%) }\end{array}$ \\
\hline Peanut paste & 55.0 & 55.0 \\
Salad oil & 0.0 & 21.0 \\
Salt & 1.0 & 1.0 \\
Sugar (granulated) & 6.2 & 6.2 \\
Cream & 5.0 & 5.0 \\
Emulsifier (sugar-ester; HLB:15) & 2.0 & 0.5 \\
Emulsifier (glyceryl monostearate; HLB:4) & 0.0 & 2.0 \\
Xantan gum & 0.0 & 0.5 \\
Water & 30.8 & 8.8 \\
\hline
\end{tabular}

water were added, stirred, and heated at $40^{\circ} \mathrm{C}$ for $5 \mathrm{~min}$. Regarding the preparation of the full-fat model peanut butter, the emulsifiers were mixed with peanut paste and salad oil in an aluminium pot at $30^{\circ} \mathrm{C}$ with later addition of cream by stirring. Sugar and salt solubilised in water were added, stirred, and heated at $40^{\circ} \mathrm{C}$ for $5 \mathrm{~min}$. As for the reduced-fat peanut butter with $\gamma$-Glu-Val-Gly, $\gamma$-GluVal-Gly was added by dissolving in water with sugar and salt. Prepared peanut butter samples were packed in glass bottles and stored at $4^{\circ} \mathrm{C}$ until sensory evaluation.

\section{Selection of the sensory panel}

In this study, 29 panellists (17 men and 12 women; $28.8 \pm$ 5.0 years old, mean \pm standard deviation) participated in the sensory evaluation. All panellists were the employees of Ajinomoto Shanghai Food Research and Technology Center and were working on the development of foods. They were Chinese and were residents of Shanghai city. In addition, all of them passed the sensory panel examination conducted using a previously described method [14]. For the comparison between the reduced-fat peanut butter and full-fat model, 20 panellists ( 9 men and 11 women; $27.6 \pm 3.6$ years old, mean \pm standard deviation) participated in the sensory evaluation. For the investigation of the effect of $\gamma$-Glu-Val-Gly, 19 panellists (13 men and 6 women; $29.9 \pm 5.3$ years old, mean \pm standard deviation) participated in the evaluation.

\section{Selection of the sensory attributes}

Panellists evaluated samples of the reduced-fat peanut butter and full-fat peanut butter model. A panel leader led the group in discussion on the differences and similarities between the samples. They developed a list of sensory attributes that described the sensory characteristics of the products. The panellists developed ten attributes: peanut flavour, saltiness, sweetness, bitterness, thick flavour, aftertaste, continuity of taste, smoothness, viscosity and oiliness. The panellists practiced rating the samples on the list so that they were prepared to begin data collection.

\section{Procedure for sensory evaluation}

The sensory evaluation was conducted between 10:00 am and 11:30 am in the partitioned booth at $25^{\circ} \mathrm{C}$ in an airconditioned sensory evaluation room. For evaluation of the peanut butter samples, $10 \mathrm{~g}$ of the sample was spread on one piece of bread $(10 \mathrm{~g})$, which was cut into four pieces. The panellists held each piece of bread with peanut butter in the mouth, evaluated the taste, and rated each attribute. They rinsed their mouths with commercial mineral water between the samples. They completed the rating for each attribute on a three-point linear scale; -1.0 : apparently weaker than the control; 0 : same as the control; and 1.0: apparently stronger than the control. For 
comparison between the reduced-fat sample and full-fat model, half of the panellists evaluated the full-fat model using a reduced-fat sample as the control and the other half evaluated the reduced-fat sample using a full-fat model as the control. Combination of the samples was randomised and balanced. Human sensory analyses were conducted following the spirit of the Helsinki Declaration, and informed consent was obtained from all panellists. The experimental procedure was approved by the Ethics Board of the Institute of Food Sciences and Technologies, Ajinomoto.

\section{Statistical analysis}

Statistical analysis was conducted using JMP version 9.0 (SAS Institute, Cary, NC, USA). The data were collected as the means \pm standard error. Data were assessed by the paired $t$-test. The data were considered to be significant at $p<0.05$.

\section{Abbreviations \\ Y-Glu-Val-Gly: $\gamma$-Glutamyl-valyl-glycine; GSH: glutathione; CaSR: calcium- sensing receptor; FEMA: Flavour and Extract Manufacturers Association; JECFA: The Joint FAO/WHO Expert Committee.}

\section{Competing interests}

The authors declare that they have no competing interests.

\section{Authors' contributions}

MK, NH and TK conceived this study. SJ and NM designed the detail of experiments. SJ conducted sample preparation and sensory evaluation. SJ and MK conducted sensory data analysis, and MK wrote the manuscript. All authors read and approved the final manuscript.

\section{Acknowledgements}

We sincerely thank Dr. Kiyoshi Miwa and Dr. Yuzuru Eto of Ajinomoto Co. Inc. for their encouragement and continued support of this work. We are grateful to Mr. Hiroaki Nagasaki, Mr. Toshifumi Imada, Mr. Takaho Tajima, Mr. Shuichi Jo, Mr. Keita Sasaki, and Ms. Takako Hirose of Ajinomoto Co. Inc., for their assistance. There is no funding in the present study.

\section{Author details}

${ }^{1}$ Institute of Food Research and Technologies, Ajinomoto Co. Inc., 1-1 Suzuki-cho, Kawasaki-ku, Kawasaki, Kanagawa 210-8681, Japan. ${ }^{2}$ Institute for Innovation, Ajinomoto Co. Inc., 1-1 Suzuki-cho, Kawasaki-ku, Kawasaki, Kanagawa 210-8681, Japan.

Received: 11 September 2014 Accepted: 20 November 2014 Published: 26 January 2015

\section{References}

1. Ohsu T, Amino Y, Nagasaki H, Yamanaka T, Takeshita S, Hatanaka T, Maruyama Y, Miyamura N, Eto Y: Involvement of the calcium-sensing receptor in human taste perception. J Biol Chem 2010, 285:1016-1022.

2. Ueda Y, Sakaguchi M, Hirayama K, Miyajima R, Kimizuka A: Characteristic flavor constituents in water extract of garlic. Agric Biol Chem 1990, 54:163-169.

3. Ueda Y, Yonemitsu M, Tsubuku T, Sakaguchi M, Miyajima R: Flavor characteristics of glutathione in raw and cooked foodstuffs. Biosci Biotech Biochem 1997, 61:1977-1980.

4. Ueda Y, Tsubuku T, Miyajima R: Composition of sulfur-containing components in onion and their flavor characters. Biosci Biotech Biochem 1994, 61:108-110.

5. Dunkel A, Koster J, Hofmann T: Molecular and sensory characterization of Y-glutamyl peptides as key contributors to the kokumi taste of edible beans (Phaseolus vulgaris L.). J Agric Food Chem 2007, 55:6712-6719.
6. Toelstede S, Dunkel A, Hofmann T: A series of kokumi peptides impart the long-lasting mouthfulness of matured Gouda cheese. J Agric Food Chem 2009, 57:1440-1448.

7. Toelstede $\mathrm{S}$, Hofmann T: Kokumi-active glutamyl peptides in cheeses and their biogeneration by Penicillium roquefortii. J Agric Food Chem 2009, 57:3738-3748.

8. Nishimura T, Egusa A: Classification of compounds enhancing "koku" to foods and the discovery of a novel "koku"-enhancing compound. Jpn J Taste Smell Res 2012, 19:167-176.

9. Flett KL, Duizer LM, Goff D: Perceived creaminess and viscosity of aggregated particles of casein micelles and k-carrageenan. J Food Sci 2010, 75:S255-S261.

10. Tomaschunas M, Zorb R, Fischer J, Kohn E, Hinrichs J, Busch-Stockfisch M: Changes in sensory properties and consumer acceptance of reduced fat pork Lyon-style and liver sausages containing inulin and citrus fiber as fat replacers. Meat Sci 2013, 95:629-640.

11. Pimentel $\mathrm{TC}$, Cruz AG, Prudencio SH: Influence of long-chain inulin and Lactobacillus paracasei subspecies paracasei on the sensory profile and acceptance of a traditional yogurt. J Dairy Sci 2013, 96:6233-6241.

12. Liou BK, Grun IU: Effect of fat level on the perception of five flavor chemicals in ice cream with or without fat mimetics by using a descriptive test. J Food Sci 2007, 72:S595-S604.

13. Amelia I, Drake MA, Nelson B, Barbano DM: A new method for the production of low-fat Cheddar cheese. J Dairy Sci 2013, 96:4870-4884.

14. Furukawa H: The Sensory Test and the Selection of Panelists, Proc. 7th Symp. Sensory Inspection; 1977:111.

doi:10.1186/2044-7248-4-16

Cite this article as: Miyamura et al:: Flavour improvement of reduced-fat peanut butter by addition of a kokumi peptide, $\gamma$-glutamyl-valyl-glycine. Flavour 2015 4:16.

\section{Submit your next manuscript to BioMed Central and take full advantage of:}

- Convenient online submission

- Thorough peer review

- No space constraints or color figure charges

- Immediate publication on acceptance

- Inclusion in PubMed, CAS, Scopus and Google Scholar

- Research which is freely available for redistribution 